\title{
Advancement of SARS-CoV-2 infection in children
}

\author{
Jacek Tabarkiewicz' , Paulina Frączek², Guoqian Chen³ ${ }^{3}$ Artur Mazur ${ }^{4}$, Jieming Wang ${ }^{5}$, \\ Fuyong Jiao ${ }^{5}$
}

\author{
'Department of Human Immunology, Institute of Medical Sciences, College of Medical Sciences, University of Rzeszow, \\ Rzeszow, Poland \\ ${ }^{2}$ Student Circle of Immunology, Institute of Medical Sciences, College of Medical Sciences, University of Rzeszow, Rzeszow, \\ Poland \\ ${ }^{3}$ Xi'an Medical University, Xi'an, Shanxi, China \\ ${ }^{4}$ Department of Pediatrics, Pediatric Endocrinology and Diabetes, University of Rzeszow, Rzeszow, Poland \\ ${ }^{5}$ Department of Pediatrics, Shanxi Provincial People's Hospital, Xi'an, Shanxi, China
}

\section{ABSTRACT}

Novel coronavirus disease 2019 (COVID-19) is a disease characterized by respiratory symptoms caused by novel coronavirus (SARS-CoV-2). Its pathogenesis is suggested to be related with ACE2 receptors, present in various organs. Children, as a special group of patients with COVID-19, have their own unique characteristics in terms of the disease's course. The majority of children present with asymptomatic course or have mild symptoms. In severe cases COVID-19 may manifest as a multisystem inflammatory syndrome; thus, correct identification of children at risk is particularly important in the diagnostic process. The treatment options for this disease are still based on general therapy, and related drug therapy is still at the clinical trial stage.

KEY WORDS:

SARS-CoV-2, COVID-19, children, multisystem inflammatory syndrome.

\section{INTRODUCTION}

Novel corona virus disease 2019 (COVID-19) is a disease characterized by respiratory symptoms caused by novel coronavirus (SARS-CoV-2). SARS-CoV-2 belongs to the genus Coronavirus, which is enveloped, often polymorphic, with round or oval-shaped particles, and a diameter of $60-140 \mathrm{~nm}$. When isolated and cultured in vitro, neo-coronaviruses can be found for about 96 hours in human respiratory epithelial cells, while isolation and culture in Vero-E6 and Huh-7 cell lines lasts approximately 6 days. The virus is UV and heat sensitive (exposure to $56^{\circ} \mathrm{C}$ for 30 minutes). Ether, $75 \%$ ethanol, aluminium-containing disinfectants, peroxyacetic acid, and chloroform can effectively inactivate the virus, but chlorhexidine is not effective in inactivating the virus [1].
The current study suggests that the mechanism of SARS-CoV-2-induced disease is related to ACE2 receptor binding [2]. ACE2 acts as a cellular receptor and binds to the viral spiking protein of SARS-CoV-2, allowing the serine protease TMPRSS2 to initiate S Protein pathogenesis $[3,4]$. The ACE2 receptor is present in various organs, especially in the lungs, heart, kidneys, gastrointestinal tract, and vascular endothelium [5-7]. As a result, children have a variety of symptoms that affect multiple systems.

\section{EPIDEMIOLOGY}

In a new epidemiological study of childhood neo-coronary pneumonia in China [8], a total of 728 (34.1\%) laboratory-confirmed cases and 1407 (65.9\%) suspected cases were reported, with a median age of 7 years

\section{ADDRESS FOR CORRESPONDENCE:}

Jacek Tabarkiewicz, Department of Human Immunology, Institute of Medical Sciences, College of Medical Sciences, University of Rzeszow, Rzeszow, Poland, e-mail: jacek.tabarkiewicz@gmail.com 
(interquartile range: 2 -13 years), comprising 1208 cases (56.6\%) in boys, and the mean time from onset to diagnosis was 2 days (range: $0-42$ days). More than $90 \%$ of all infected patients were asymptomatic, with mild or moderate course. Children of all ages were COVID-19 sensitive, and no significant gender differences were found. The clinical manifestations of COVID-19 cases in children are usually less severe than those in adult patients, but young children (especially infants) are vulnerable to infection, which may be related to the fact that children are immunocompromised and that ACE2 in children may not be as developed as in adults.

SARS-CoV-2 spreads primarily through respiratory droplets and close contact [9], and transmission may also be facilitated by prolonged exposure to high concentrations of aerosols in relatively confined environments; more specifically, the airtightness of the environment and the density of virus per unit volume affect the transmission of SARS-CoV-2.

Human mucosal cells express ACE2 receptors, which are abundant in air-exposed lips, eyelids, nasal cavities, lung cells, etc. [10]. Therefore, in addition to respiratory transmission, infectious droplets and body fluids can contaminate the human conjunctival epithelium. Respiratory viruses can also lead to ocular complications in infected patients, which in turn can lead to respiratory infections. Thus, exposure to SARS-CoV-2 can cause acute respiratory infections through multiple pathways $[11,12]$.

Wang et al. suggested the possibility of mother-tochild transmission of SARS-CoV-2 based on the positive nasopharyngeal swab of SARS-CoV-2 nucleic acid test 36 hours after the birth of a newborn with confirmed COVID-19 delivery [13]. However, Chen et al. also reported that 9 pregnant women with a diagnosis of COVID-19, who underwent caesarean section, had cord blood, amniotic fluid, breast milk, and neonatal oropharyngeal swab negative for SARS-CoV-2 nucleic acid [14]. Therefore, whether there is vertical transmission of SARS-CoV-2 from mother to child is still debatable, and more evidence is needed to clarify this topic.

Cai et al. showed that SARS-CoV-2 has a high detection rate of viral RNA in children's faeces, with a detoxification period of up to 2-4 weeks, suggesting that the disease may be faecal-oral transmitted [15]. However, because the study did not capture live virus, there is no direct evidence of faecal-oral transmission. Xu et al. performed nucleic acid testing on anal swabs from 10 children with COVID-19 and found that positive anal swabs and prolonged intestinal detoxification may be more useful than the nasopharyngeal swab test in determining treatment efficacy and quarantine termination time [16].

\section{CLINICAL MANIFESTATIONS}

Children with COVID-19 are most frequently affected by mild or asymptomatic manifestation of disease [17]. It may be accompanied by fatigue, myalgia, nasal congestion, runny nose, headache, and dizziness. A small group of children do not have fever and present only with cough or diarrhoea [18]. Some children and newborns have atypical symptoms, with vomiting, diarrhoea, and other gastrointestinal symptoms as the first manifestation, or only asthma and shortness of breath $[19,20]$. In severe cases rapid progress to respiratory distress syndrome, septic shock, uncorrectable metabolic acidosis, coagulopathy [21], and multiple organ failure may occur [22].

In severe cases, some children can present with multisystem inflammatory syndrome [23, 24]. Many of them have fever and cutaneous or mucosal manifestations similar to Kawasaki disease [25-27]. Some exhibit features of toxic shock syndrome, secondary phagocytic lymphohistiocytosis, or macrophage activation syndrome [28-30]. In a retrospective analysis of 186 children with MIS-C (multisystem inflammatory syndrome in children) in the United States [31], 109 patients (59\%) had incomplete or failing respiratory function, and coronary aneurysm was found in $8 \%$ of the patients $(15 / 186)$ and in $9 \%(15 / 170)$ of those with echocardiograms. The majority of these 186 children ( $71 \%$ in 132 cases) showed involvement of at least 4 organ systems. The most commonly affected organ systems were the gastrointestinal system (171 [92\%]), cardiovascular system (149 [80\%]), haematological system (142 [76\%]), the mucocutaneous system (137 [74\%]), and the respiratory system (131 [70\%]).

\section{LABORATORY TEST FINDINGS}

Children usually have normal or elevated white blood cell counts and normal or elevated CRP and PCT. Studies have shown that $80 \%-95 \%$ of patients have elevated CRP [31]. Unlike adults, children usually do not have lymphopaenia and have normal white blood cell counts. In severe cases, children may have elevated liver enzymes and cardiac enzymes, and the current preferred method for detecting viral RNA is RT-PCR.

\section{RADIOGRAPHIC EXAMINATION}

Chest CT can show characteristic changes of subpleural gross glass clouding and peripheral halo solids and is an effective tool for follow-up and evaluation of changes in lung pathology [32]. In the case of pharyngeal swab specimens with a low positive COVID-19 nucleic acid test rate, early detection of lesions by CT is beneficial to the child's rational and early treatment [33]. However, it has little role in assessing the clinical rehabilitation of children.

\section{DIAGNOSIS}

The diagnosis is confirmed on the basis of the child's history of epidemiologic exposure, symptoms, laboratory 
TABLE 1. Severity of NCP according to clinical and radiological features

\begin{tabular}{|l|l|}
\hline Severity & Clinical and radiological features \\
\hline Mild & Mild clinical symptoms, no signs of pneumonia on imaging \\
\hline Moderate & With fever, respiratory symptoms, and pneumonia visible on imaging \\
\hline Severe & $\begin{array}{l}\text { Any of the following: } \\
\text { 1. The occurrence of shortness of breath, except for the effects of fever and crying. } \\
\text { 2. In the resting state, an oxygen saturation level less than or equal to 92\%. } \\
\text { 3. Assisted breathing (moaning, nasal firings, triple concave sign), cyanosis, intermittent apnea. } \\
\text { 4. Appearance of lethargy, seizures. } \\
\text { 5. Refusal to eat or difficulty feeding, signs of dehydration. }\end{array}$ \\
\hline Critical & $\begin{array}{l}\text { 1. A child with respiratory failure requiring mechanical ventilation. } \\
\text { 2. Children who are in shock. } \\
\text { 3. Children with other organ failure requiring ICU monitoring and treatment. }\end{array}$ \\
\hline
\end{tabular}

tests, influential features of chest CT, positive nucleic acid detection by PT-PCR for novel coronavirus, or viral gene sequencing with high homology to known new coronaviruses and neo-coronavirus-specific antibody testing [34].

According to China's novel coronavirus pneumonia treatment protocol ( $7^{\text {th }}$ edition in trial), the severity of the disease is categorized into 4 levels: mild, moderate, severe, and critical (Table 1) [1]. Early identification of high-risk children is particularly important given the special characteristics of this group. It is important to identify the child at risk promptly and recognize the child with a rapid respiratory rate, impaired consciousness, lethargy, progressively elevated lactate on laboratory tests, bilateral or multiple enlarged lymph nodes on radiographs, and general high risk of developing the disease. Infiltrates, pleural effusions or short-term rapidly progressing lesions, infants under 3 months of age, or with underlying conditions such as congenital heart or bronchi disease are other high-risk factors. Children with dysplasia, respiratory malformations, severe malnutrition, who are immunocompromised, or who have been using immunosuppressants for a long time may rapidly progress to critical illness $[1,35,36]$. Zhu et al. consider neonates who meet the following criteria to be at high risk for SARS-CoV-2 infection [37]:

1. The mother is at high risk before delivery- persistent fever during or after childbirth, routine laboratory tests showing decreased lymphocyte ratio.

2. Chest CT showing pulmonary infiltrates. Viral nucleic acid test results and epidemiological history suggest that the mother is a confirmed/highly suspected case (exclude other viral and bacterial infections, mycoplasma infections, postpartum fever, breast swelling, mastitis, and other obstetric diseases).

3. Clinical manifestations or epidemiology in the mother. Neonates with a history of infection but in whom the level of suspicion is not sufficient to confirm the diagnosis.

4. Neonates with an epidemiological history of infection, including community and obstetric sources, family members or caregivers of mother-infant co-housed sourc- es, and visitors with diagnosis/high suspicion of SARSCoV-2 infection.

5. Neonates living in or visiting environments at high risk of SARS-CoV-2 infection.

\section{TREATMENT}

The current treatment option is a general treatment, such as bed rest, enhanced nutrition and child psychological relief treatment, etc., which can be extended to effective oxygen therapy measures if necessary. Highflow oxygen and non-invasive mechanical ventilation can be given when the child is in respiratory failure and ARDS, and invasive mechanical ventilation can be used in children with respiratory failure and ARDS. If the pulmonary ventilation strategy fails, it may be switched to extracorporeal membranes oxygenation (ECMO) symptomatic support therapy [36]. In terms of pharmacotherapy, the use of anticoagulation therapy is recommended in patients with early-stage COVID-19, especially when the $\mathrm{D}$-dimer value is 4 times higher. The presence of inflammation and other disease-related factors can cause overactivation of coagulation, thereby increasing the risk of ischaemic events and disseminated intravascular coagulation (DIC) [37]. The use of antiviral drugs in the treatment of COVID-19 follows the experience with SARS$\mathrm{CoV}$ and MERS-CoV. There are limited data on the use of antiviral drugs in children. Remdesivir has recently received emergency FDA authorization for use in hospitalized adults and paediatric patients aged $<12$ years. It also received emergency use authorization for hospitalized paediatric patients weighing 3.5 to $40 \mathrm{~kg}$ or $<12$ years of age and weighing $\geq 3.5 \mathrm{~kg}$, for compassionate use [38]. The COVID-19 Treatment Guidelines Panel recommends the use of remdesivir to treat hospitalized patients with $\mathrm{SpO}_{2} \leq 94 \%$ on ambient air or those who require supplemental oxygen. The panel recommends a 5-day course for non-intubated patients. Data are insufficient, but for patients on mechanical ventilation or ECMO, experts recommend a 10-day course [39]. On 21 November 2020, the FDA issued emergency use authorization for 
the monoclonal antibodies casirivimab and imdevimab to be administered together, for the treatment of mild to moderate COVID-19 in adults and children aged $<12$ years and weighing at least $40 \mathrm{~kg}$, who are at high risk for progressing to severe COVID-19 disease. [40, 41].

\section{CONCLUSIONS}

Children, as a relatively special group, have milder or even asymptomatic course of disease after infection with SARS-CoV-2 as opposed to adults. Many children start with little or no associated respiratory signs, and it is important to focus on extra-pulmonary symptoms such as gastrointestinal signs in such children to screen for SARS$\mathrm{CoV}-2$ infection. Although critically ill children are less common than adults, the symptoms may be more severe and may involve multiple systems resulting in multiple corresponding clinical manifestations. Especially important for the prognosis is early diagnosis and treatment of children with certain basic diseases. It is crucial to recognize the Kawasaki-like features and MIS-C symptoms in children, to distinguish them from other diseases, and to set the proper diagnosis of SARS-CoV-2 infection early and to implement appropriate measures.

Given that the world is still in the process of understanding and exploring the new coronary pneumonia, especially the drug treatment field, there are still no clear and effective treatment regimes. Research data on COVID-19 in children is far less advanced than in adults, and more evidence and research are needed to further understand the impact of the disease on children.

\section{DISCLOSURE}

The authors declare no conflict of interest.

\section{REFERENCES}

1. Diagnosis and Treatment of New Coronavirus Pneumonia (Trial Version 7). Chinese Medicine 2020; 15: 801-805.

2. South AM, Brady TM, Flynn JT. ACE2 (Angiotensin-Converting Enzyme 2), COVID-19, and ACE Inhibitor and Ang II (Angiotensin II) Receptor Blocker Use During the Pandemic: The Pediatric Perspective. Hypertension 2020; 76: 16-22.

3. Liu H, Gai S, Wang X, et al. Single-cell analysis of SARS-CoV-2 receptor ACE2 and spike protein priming expression of proteases in the human heart. Cardiovasc Res 2020; 116: 1733-1741.

4. Dehelean CA, Lazureanu V, Coricovac D, et al. SARS-CoV-2: Repurposed Drugs and Novel Therapeutic Approaches-Insights into Chemical Structure-Biological Activity and Toxicological Screening. J Clin Med 2020; 9: 2084.

5. Chi X, Yan R, Zhang J, et al. A neutralizing human antibody binds to the N-terminal domain of the Spike protein of SARS-CoV-2. Science 2020; 369: 650-655.

6. Castagnoli R, Votto M, Licari A, et al. Severe Acute Respiratory Syndrome Coronavirus 2 (SARS-CoV-2) Infection in Children and Adolescents: A Systematic Review. JAMA Pediatr 2020; 174: 882-889.
7. Wrapp D, Wang N, Corbett KS, et al. Cryo-EM structure of the 2019-nCoV spike in the prefusion conformation. Science 2020; 367: 1260-1263.

8. Dong Y, Mo X, Hu Y, et al. Epidemiology of COVID-19 Among Children in China. Pediatrics 2020; 145: e20200702.

9. Zhang $\mathrm{H}$, Kang Z, Gong $\mathrm{H}$, et al. The digestive system is a potential route of 2019-nCov infection: a bioinformatics analysis based on single-cell transcriptomes. bioRxiv, 2020.

10. Lu CW, Liu XF, Jia ZF. 2019-nCoV transmission through the ocular surface must not be ignored. Lancet 2020; 395: e39.

11. Xia J, Tong J, Liu M, et al. Evaluation of coronavirus in tears and conjunctival secretions of patients with SARS-CoV-2 infection. J Med Virol 2020; 92: 589-594.

12. Zhang H, Kang Z, Gong H, et al. The digestive system is a potential route of 2019-nCov infection: a bioinformatics analysis based on single-cell transcriptomes. bioRxiv 2020; DOI: https://doi. org/10.1101/2020.01.30.927806.

13. Wang S, Guo L, Chen L, et al. A case report of neonatal 2019 coronavirus disease in China. Clin Infect Dis 2020; 71: 853-857.

14. Chen H, Guo J, Wang C, et al. Clinical characteristics and intrauterine vertical transmission potential of COVID-19 infection in nine pregnant women: a retrospective review of medical records. Lancet 2020, 395: 809-815.

15. Jiehao C, Jin X, Daojiong L, et al. A case series of children with 2019 novel coronavirus infection: clinical and epidemiological features. Clin Infect Dis 2020; 71: 1547-1551.

16. Wang W, Xu Y, Gao R, et al. Detection of SARS-CoV-2 in Different Types of Clinical Specimens. JAMA 2020; 323: 1843-1844.

17. Yaoling M, Shengying X, Min W, et al. Analysis of clinical features of 115 children infected with new coronavirus. Chinese Journal of Contemporary Pediatrics 2020; 22: 290-293.

18. Mantovani A, Rinaldi E, Zusi C, et al. Coronavirus disease 2019 (COVID-19) in children and/or adolescents: a meta-analysis. Pediatr Res 2020; 89: 733-737.

19. Meena J, Yadav J, Saini L, et al. Clinical Features and Outcome of SARS-CoV-2 Infection in Children: A Systematic Review and Meta-analysis. Indian Pediatr 2020; 57: 820-826.

20. Ye Q, Wang B, Zhang T, et al. The mechanism and treatment of gastrointestinal symptoms in patients with COVID-19. Am J Physiol Gastrointest Liver Physiol 2020; 319: G245-G252.

21. Xingfeng Ch, Furong Z, Ruihua X, et al. Cases of disseminated intravascular coagulation in 2 infants with novel coronavirus pneumonia. Chinese Journal of Integrated Chinese and Western Medicine 2020; 27: 46-49.

22. Feng Ch, Zhisheng L, Furong Z, et al. China's First New Critical Coronavirus Pneumonia in Children. Chinese Journal of Pediatrics 2020; 58: 179-182.

23. Riphagen S, Gomez X, Gonzalez-Martinez C, et al. Hyperinflammatory shock in children during COVID-19 pandemic. Lancet 2020; 395: 1607-1608.

24. Dufort EM, Koumans EH, Chow EJ, et al. Multisystem Inflammatory Syndrome in Children in New York State. N Engl J Med 2020; 383: 347-358.

25. Belhadjer Z, Méot M, Bajolle F, et al. Acute heart failure in multisystem inflammatory syndrome in children (MIS-C) in the context of global SARS-CoV-2 pandemic. Circulation 2020; 142: 429-436.

26. Verdoni L, Mazza A, Gervasoni A, et al. An outbreak of severe Kawasaki-like disease at the Italian epicentre of the SARS-CoV-2 epidemic: an observational cohort study. Lancet 2020; 395: 1771-1778.

27. Roe K. A viral infection explanation for Kawasaki disease in general and for COVID-19 virus-related Kawasaki disease symptoms. Inflammopharmacology 2020; 28:1219-1222.

28. Ouldali N, Pouletty M, Mariani P, et al. Emergence of Kawasaki disease related to SARS-CoV-2 infection in an epicentre of the French 
COVID-19 epidemic: a time-series analysis. Lancet Child Adolesc Health 2020; 4: 662-668.

29. Toubiana J, Poirault C, Corsia A, et al. Kawasaki-like multisystem inflammatory syndrome in children during the covid-19 pandemic in Paris, France: prospective observational study. BMJ 2020; 369: m2094.

30. MacDonald KL, Osterholm MT, Hedberg CW, et al. Toxic shock syndrome: a newly recognized complication of influenza and influenzalike illness. JAMA 1987; 257: 1053-1058.

31. Feldstein LR, Rose EB, Horwitz SM, et al. Multisystem Inflammatory Syndrome in U.S. Children and Adolescents. N Engl J Med 2020; 383: 334-346.

32. Huang W, Berube J, McNamara M, et al. Lymphocyte Subset Counts in COVID-19 Patients: A Meta-Analysis. Cytometry A 2020; 97: 772-776.

33. Zhen-Dong Y, Gao-Jun Z, Run-Ming J, et al. Clinical and transmission dynamics characteristics of 406 children with coronavirus disease 2019 in China: A review. J Infect 2020; 81: e11-e15.

34. Xia W, Shao J, Guo Y, et al. Clinical and CT features in pediatric patients with COVID-19 infection: Different points from adults. Pediatr Pulmonol 2020; 55: 1169-1174.

35. Ma H, Hu J, Tian J, et al. A single-center, retrospective study of COVID-19 features in children: a descriptive investigation. BMC Med 2020; 18: 123.

36. Liu H, Gai S, Wang X, et al. Single-cell analysis of SARS-CoV-2 receptor ACE2 and spike protein priming expression of proteases in the human heart. Cardiovasc Res 2020; 116: 1733-1741.

37. Lin L, Lu L, Cao W, Li T. Hypothesis for potential pathogenesis of SARS-CoV-2 infection - a review of immune changes in patients with viral pneumonia. Emerg Microbes Infect 2020; 9: 727-732.

38. Remdesivir. COVID-19 Treatment Guidelines. Accessed December11, 2020.https://www.covid19treatmentguidelines.nih.gov/antiviral-therapy/remdesivir/.

39. Al-Tawfiq JA, Al-Homoud AH, Memish ZA. Remdesivir as a possible therapeutic option for the COVID-19. Travel Med Infect Dis 2020; 34: 101615

40. Casirivimab. In: Drugs and Lactation Database (LactMed). National Library of Medicine (US); 2006. Accessed December 14, 2020. http://www.ncbi.nlm.nih.gov/books/NBK564279/.

41. Statement on Casirivimab Plus Imdevimab EUA. COVID-19 Treatment Guidelines. Accessed December 14, 2020. https://www.covid19treatmentguidelines.nih.gov/statement-on-casirivimab-plusimdevimab-eua/. 